\title{
Política de las pedagogías en la construcción del oficio. La formación situada de investigadores en ciencias sociales
}

\author{
Pedagogy policy in the construction of the work. Social sciences researchers' \\ situated training
}

\author{
María Belén Álvaro \\ mabalvaro@yahoo.com.ar \\ Facultad de Derecho y Ciencias Sociales, Universidad \\ Nacional del Comahue, Argentina

\section{Delfina Garino} \\ delgarino@gmail.com \\ Facultad de Derecho y Ciencias Sociales, Universidad \\ Nacional del Comahue. Instituto Patagónico de \\ Estudios de Humanidades y Ciencias Sociales \\ (IPEHCS-UNCo-CONICET), Argentina
}

Cita sugerida: Álvaro, M. B. y Garino, D. (2020). Política de las pedagogías en la construcción del oficio. La formación situada de investigadores en ciencias sociales. Revista Latinoamericana de Metodología de las Ciencias Sociales, 10(2), e078. https://doi.org/10.24215/18537863e078

Recepción: 03 Febrero 2020

Aprobación: 27 Agosto 2020

Publicación: 01 Diciembre 2020
Resumen: Este trabajo constituye una instancia de sistematización del devenir de nuestros modos posibles de construir y formar en la investigación social en la etapa avanzada de la carrera de grado de Sociología. Las autoras de este artículo somos docentes del área de metodología de las ciencias sociales de la carrera de Sociología en la Universidad Nacional del Comahue. Las indagaciones que se proponen les estudiantes nos interpelan hacia problematizaciones sobre nuestras propias trayectorias, esquemas conceptuales y posicionamientos teóricopolíticos, que se consolidan en estos años en el pasaje por un triple giro epistémico cuyas derivas inasibles interpelan y trasmutan los modos posibles de hacer el oficio. Desde estos posicionamientos, proponemos reflexionar sobre cómo se transmite un hacer, en un ejercicio constante de vigilancia epistemológica, que reconozca provisionalmente un sistema de esquemas siempreen-permanente-sospecha, y sobre la construcción de una idea de validez que implica una doble rigurosidad, que es de coherencia interna y validación teórico-política externa.

Palabras clave: Pedagogías, Epistemología, Formación, Investigación social.

Abstract: This article systematizes the evolution of our possible strategies of doing and teaching social research in Sociology degree career. We are teachers of the area of methodology of social sciences at Comahue National University. The researches that students proposes, challenge us to problematizations about our own trajectories, conceptual schemes and theoreticalpolitical positions, which has been consolidated in recent years in the passage through a triple epistemic turn, whose elusive drifts challenge and transmute the possible ways of doing the craft of sociology. From these positions, we propose to reflect on how the craft is built and transmitted, from a constant exercise of epistemological vigilance, which provisionally recognizes a system of always-in-permanent-suspicion schemes. We also propose to build an idea of validity that implies a double rigor of internal coherence and external theoretical-political validation.

Keywords: Pedagogies, Epistemology, Training, Social research. 


\section{INTRODUCCIÓN: (RE)PROGRAMANDO LAS CONDICIONES DE POSIBILIDAD DEL PENSAMIENTO SITUADO}

Este trabajo constituye una instancia de reflexión acerca de la formación de investigadores en la carrera de grado de Sociología, a partir de devenires epistemológicos ineludibles y los saberes-prácticas de investigación que promovemos, desarrollamos y habilitamos a les estudiantes.

Las autoras de este artículo somos docentes del área de metodología de las ciencias sociales de la carrera de Sociología en la Universidad Nacional del Comahue. Nos encontramos dictando desde hace siete años una asignatura de cursada anual y correspondiente $\mathrm{al}^{\circ}$ año de la Licenciatura. Es correlativa de tres materias cuatrimestrales que componen las Metodologías de las Ciencias Sociales y es previa al Taller de Tesis.

Dado que sólo desde la enseñanza, y en tanto recurso pedagógico, ha sido posible separar la tríada Epistemología-Teoría-Metodología, proponemos el Seminario de Formación de Investigadores (SFI) como una instancia de re-unión de estos componentes, resignificada por la práctica situada que requiere una investigación concreta.

Tomando el lenguaje como "estratégico campo de batalla, sitio de pugnas en torno a los modelos de (in)inteligibilidad del mundo, de los mundos” (Flores, 2013, p. 79), la formación en la investigación se trata, para nosotras, de una auto-reflexividad sobre las prácticas del conocer, que proporciona elementos críticos para el uso de las categorías y esquemas de pensamiento.

Así, recuperamos la política de investigación como toma de posición en el mundo y encaramos una acción pedagógica de acompañamiento teórico-práctico para la formación en el oficio desde el intercambio horizontal y recíproco.

La organización de la cursada en grupos de investigación propone recuperar la impronta colectiva de la producción social de conocimiento. Promovemos formas de concebir y llevar adelante investigaciones sociales que supongan el proceso de investigación como una acción política situada. Por este motivo, las estrategias metodológicas y de análisis se van construyendo artesanalmente en función de los problemas de investigación que les estudiantes elaboran, en íntima vinculación con una práctica de co-producción con otres a la que nunca anteceden ni determinan su contenido.

Nuestra apuesta política consiste en batallar contra la elitista idea de que "investigar no es para todo el mundo", y presentar la investigación como un oficio que, al ejercerlo, humaniza y politiza el quehacer sociológico.

El "oficio" es para Bourdieu (2002) un ejercicio constante, a partir de un sistema de esquemas más o menos dominados y más o menos trasponibles, y de la vigilancia epistemológica que subordine el uso de técnicas y conceptos a un examen sobre las condiciones y los límites de su validez. En ese sentido, como docentes e investigadoras nos preguntamos ¿̨cómo se transmite un hacer, un ejercicio constante de vigilancia epistemológica, si no es bajo la impronta de reconocer provisoriamente un sistema de esquemas siempre-enpermanente-sospecha? ¿Cómo se construye una idea de validez si no es a partir de una doble rigurosidad que es de coherencia interna y validación teórico-política externa?

A lo largo de estos años hemos logrado acompañar potentes trabajos que han conducido a ricas producciones sobre temáticas concretas que configuran "afectos" (emociones vitales) tanto para quienes las proponen como punto de indagación, como para quienes las transitan de manera vital y son convocades a ser parte del recorte de investigación en territorios relacionales temporales, variables y variados (Rolnik, 2019).

Las indagaciones que se proponen les estudiantes nos interpelan hacia problematizaciones sobre nuestras propias trayectorias, esquemas conceptuales y posicionamientos teórico-políticos, que se consolidan en estos años en el pasaje por un triple giro epistémico cuyas derivas inasibles esbozamos a lo largo de este escrito. 


\title{
2. LA TRANSMISIÓN DEL OFICIO EN LA CARRERA DE GRADO DESDE SUBJETIVIDADES SITUADAS
}

\author{
"La lógica de un pensamiento es una ráfaga soplando sobre nosotros. Como decía Leibniz: cuando \\ creíamos haber llegado a puerto, nos encontramos de nuevo arrojados en alta mar."
}

Gilles Deleuze

Este ejercicio de sistematización del devenir de nuestros modos posibles de construir investigación social en la etapa avanzada de carrera de grado lo libramos en todos los frentes, analíticamente separados, pero vitalmente entretejidos: el epistemológico, el teórico-político y el metodológico-técnico.

A lo largo de siete años, nuestras propias prácticas de acompañamiento en la formación de investigadores han sido interpeladas por lo personal-político y por la riqueza de las miradas y trayectorias sociales de les estudiantes. Al principio, en una dialéctica que fuimos resolviendo más intuitivamente, y luego, desde la comprensión de que nuestro trabajo está inexorablemente construido desde "la contingencia histórica radical para todas las afirmaciones del conocimiento y los sujetos conocedores" para Haraway (en Figari, 2010, p.1).

Desde nuestras actuancias del oficio, posicionadas en un enfoque epistemológico crítico, proponemos a quienes transitan por la materia ejercitar un "enfoque de la sospecha" que, lejos de estar centrado en la performatividad de las técnicas, o la destreza metodológica, prioriza el posicionamiento teóricometodológico desde una pedagogía político-creativa de la formación. Entendemos esta última como punto de partida en la co-construcción de conocimiento, cuyas condiciones de posibilidad deben ser negociadas ética, horizontal y permanentemente con quienes son en conocimiento y acción en el campo (Berkin y Kaltmeier, 2012).

Partiendo de esta articulación teoría-práctica, promovemos formas de concebir y llevar adelante investigaciones sociales que supongan el proceso de investigación como un posicionamiento político en permanente esfuerzo reflexivo y autorreflexivo; y a los procedimientos y técnicas como dispositivos de resolución material de las necesidades conceptuales. A decir de Foucault (1988), el trabajo analítico no puede proceder sin una conceptualización permanente, la cual implica un pensamiento crítico, una revisión constante en la que se tengan en cuenta las condiciones históricas que la motivan.

Con cada propuesta grupal de investigación, en la cual convergen intereses individuales y colectivos, la complejidad de los interrogantes que emergen nos hace experimentar las distancias que nos anudan desde distintos puntos a una suerte común de trabajo, como desnaturalización. La importancia de abrir visibilidad y, por ende, crear condiciones de enunciabilidad académica de las dimensiones sociohistóricas de la subjetividad, permite diseñar abordajes desde criterios multirreferenciales, pensando de otro modo la relación entre "lo individual" y "lo social", buscando y desarrollando estrategias para superar las antinomias im-posibles.

Así, a lo largo de estos años hemos producido, mediante el ejercicio y transmisión del oficio, una forma de "hacer y des-hacernos" (Manada de lobxs, 2014, p. 13) que ha generado condiciones de posibilidad para nuestro pasaje crítico por tres grandes giros epistémicos, no lineales, interseccionales, como forma de politizar el oficio: postestructuralismo, decolonialidad y epistemología feminista. La metodología marxista, que formaba parte sustancial de nuestra propuesta de cátedra en los primeros años, ha sido colocada en tensión respecto de quién conoce, cómo y para qué se conoce. La pregunta permanente por la proximidad y el lugar en el proceso de conocimiento de quienes son con nosotres en la investigación ha abierto intersticios desde los cuales líneas de fuga múltiples e insistentes se condensaron, para redistribuir las zonas de atención.

Estos posicionamientos han ganado cuerpo en nuestra propuesta, pero también en nuestras prácticas investigativas y vitales, en un complejo entramado de experiencia-vivencia que impregna la mirada desde el inicio y da protagonismo al encuentro con otres como sustento del proceso de conocimiento. 


\title{
2.1- Desplazamientos epistemológicos: desde y de las preguntas concretas
}

\author{
"Los únicos que terminan creyendo y actuando según las doctrinas ideológicas de la descarnada objetividad encerrada \\ en los libros de texto elementales y en la literatura científica son los no \\ científicos y unos pocos filósofos que se lo creen todo" (Haraway, 1995, p. 317)
}

La mirada postestructuralista se hace lugar como construcción teórica de gran potencia explicativa en trabajos en los que les estudiantes se preguntan por los procesos de construcción de subjetividades en quienes participan en espacios de formación laboral en la capacitación para el oficio o en contextos de encierro. La apertura a lecturas en las que la reconceptualización de las nociones de discurso, poder y construcción de subjetividad posibilitan hacer visible la producción de regímenes de verdad en educación y formación nos devuelve la necesidad de ejercitar un auto-análisis sobre las propias prácticas de investigación que desarrollamos en nuestra tarea cotidiana.

El interés por el lenguaje como fuerza productiva constitutiva, y el poder, el deseo y la representación como categorías discursivas, permiten conducir a la inversión y renegociación de las relaciones de autoridad e incrementar las comprensiones de las dinámicas del poder, a menudo contradictorias, que suceden en las prácticas pedagógicas y que nos afectan en tanto involucrades en ellas. Abre la posibilidad de generar resistencias y producir sentidos que subviertan las prácticas pedagógicas dominantes, y de preguntarnos con Scardamaglia:

\footnotetext{
“¿Cómo evitar, en algunos momentos, las capturas de la maquinaria estupidizante? ¿Cómo no dejarse tomar por ciertas prácticas si hemos sido producides por ellas? ¿Cómo desactivar la operatoria intimidante del miedo, de la transacción, de la obligación vacía? ¿Cómo no enamorarse del poder? ¿Cómo evitar la indignidad de hablar por otres? Quizás la posibilidad de situar los dispositivos a través de los que esta maquinaria opera, nos permita, por instantes, desmontarlos. Quizás en el trabajo con otres podamos alertarnos de nuestras capturas" (Scardamaglia, 2018, p.1).
}

El giro decolonial gana condiciones de posibilidad desde la necesidad de acceder a la economía simbólica que instala un orden jerárquico y lo reproduce. Nos permite re-localizar la mirada "en el paisaje latinoamericano y desde allí situarnos en el contexto del poder globalmente hegemónico” (Segato, 2013, p. 36). Dado que, como señala Segato (2010a), es necesario escudriñar a través de las representaciones, las ideologías, los discursos, este desplazamiento habilita pensar en investigaciones en las que les estudiantes se preguntan acerca del sentido de la "interculturalidad" como forma de construcción de la currícula en escuelas de Neuquén ubicadas en territorios mapuce, o en las que la racialización de los cuerpos se torna hipótesis central en el caso del homicidio de un trabajador "golondrina” en manos de las fuerzas policiales de la provincia de Río Negro.

Asimismo, la perspectiva decolonial desentrama y dota de sentido a la incomodidad política que puede generar la mirada hacia 'la otredad' investigada, volviéndola proximidad histórica. En su propuesta, busca visibilizar y reivindicar otros modos de vivir, de significar, de construir, de ser y de hacer mundos diferentes al establecido por el programa de la modernidad occidental colonial (Sánchez Rubio, 2012). La centralidad analítica que se le atribuye en este pensamiento a la racialización y biologización de las jerarquías, y la problematización de la emergencia, consolidación y reproducción de los Estados coloniales, son algunos de los puntos centrales que anudan la perspectiva decolonial.

La disección de la matriz colonial y de la construcción de la subjetividad colonial permiten analizar la perpetuación de las dinámicas de racialización y segregación en las que se sustentan las lógicas actuales de la violencia y de la explotación extractiva a escala planetaria. Una crítica de la modernidad en la que la definición misma de la práctica colonial como "una concordancia entre la lógica de las razas y las lógicas del beneficio (...), una forma de poder constituyente por el modo que ata las poblaciones y el territorio" (Gago y Obarrio, 2016) permite desnaturalizar prácticas que homogeneizan y clasifican jerárquicamente cuerpos, pero también disciplinas, saberes, discursos, prácticas, antes excluidas de la metodología y hoy resignificadas a la luz de la problematización misma de las jerarquías del proyecto moderno. Es, al mismo tiempo, un llamado 
político a recuperar un "proyecto histórico" de los vínculos entre pueblos clausurado por el proyecto de "las cosas" propio de la colonialidad (Segato, 2013).

En síntesis, los interrogantes que nos permite abrir este posicionamiento teórico, como propone Segato, son que nuestro "objeto clásico" sea hoy el que nos interpele, nos diga quiénes somos y qué se espera de nosotros, y nos demande el uso de nuestra "caja de herramientas" para responder a sus preguntas y contribuir a su proyecto histórico; "permitir que la mirada del otro abra un juicio sobre nosotros" (Segato, 2013, p. 12). A decir de Cusicanqui, "Construir nuestra propia ciencia - en un diálogo entre nosotros mismos-, dialogar con las ciencias de los países vecinos, afirmar nuestros lazos con las corrientes teóricas de Asia y África, y enfrentar los proyectos hegemónicos del norte con la renovada fuerza de nuestras convicciones ancestrales" (Rivera Cusicanqui, 2010, p. 73).

En íntima articulación con estos movimientos epistemológicos y con los pliegues mismos de sus debates, el giro de la epistemología feminista se nos hace cuerpo en militancia y pregunta de investigación. En las propuestas de quienes cursan el seminario emergen interrogantes que refieren a cuáles son las condiciones de habitabilidad de las infancias trans en las instituciones escolares, los procesos de subjetivación política de las trabajadoras sexuales en localidades petroleras, la construcción de "política en femenino" de trabajadoras de una fábrica textil recuperada.

Aquí, la epistemología feminista que vamos encarnando nos sitúa en una mirada cuyas bases son, en palabras de Harding, más confiables para la elaboración del conocimiento (1993), ya que por debajo de la superficie de las relaciones sociales permiten desmontar estructuras de relaciones entre posiciones jerárquicamente ordenadas e interseccionalmente ligadas. De esta manera, es dable investigar diseccionando analíticamente y subvirtiendo discursivamente aquellos mecanismos que reproducen sistemas de opresión, como el sexismo y el androcentrismo, pero también clasismo y racismo, discriminación y fragilización, que operan como naturalizaciones y que forman parte de la historia larga de la humanidad, aunque con distinto nivel de intensidad (Segato, 2010b).

La epistemología feminista es aquella que reconoce e incluye la especificidad de las experiencias y de su permeabilidad al poder (Haraway, 1995). Al reconocer la parcialidad situada del conocimiento, abre la posibilidad a lecturas responsables de lo real, no totalizantes u homogeneizantes, y genuinamente enriquecedoras. Remarca que la producción de hechos científicos siempre ocurre dentro de narrativas específicas. Así, reconoce el carácter "ficcional y lingüístico-material" de la ciencia cuando la define, sobre todo, como "una práctica de contar historias", no perdiendo de vista nunca las demandas éticas y materiales planteadas por los discursos científicos: "los hechos científicos pueden ser narraciones radicalmente históricas y contingentes, pero es igualmente cierto que no todas las narraciones son iguales aquí" afirma Orr (en Haraway, 1995, p. 313).

Heredera de la política de localización de Adrienne Rich, al situar la objetividad en el reconocimiento del punto de partida del propio conocer, de sus limitaciones y su carácter contingente, Haraway logra "mostrar la operación ideológica que supone esgrimir la noción de objetividad en la ciencia” (Fígari, 2011, p. 1) y propone una auto-explicitación política del sujeto cognoscente: "los conocimientos situados crecen con la responsabilidad por articular la especificidad de la localización desde la que deben construirse la política y el conocimiento" (Haraway, 1995, p. 186). Para Harding (1993), ello supone una "reflexividad fuerte" por la cual no hay una desvinculación aséptica, sino una asunción responsable del acto de conocer (Fígari, 2010, pp. 9-10). Para dar cuenta del conocimiento situado, Haraway recupera la categoría de experiencia de Lauretis, para quien "experiencia es una semiosis, una encarnación de significados, uno de los aspectos del movimiento histórico" (Haraway, 1995, p. 184).

De este modo, la epistemología feminista nos propone trabajar desde la reflexividad fuerte del conocimiento situado, poniendo de relieve que la objetividad no dependerá de la validez de los enunciados y metodologías sino de les propies implicades en el hacer ciencia según Horkheimer (citado por Figari, 2010) 
y del abordaje de la diferencia situada a partir del abandono de la "ilusión de lo único" (Haraway, 1995, p. 209), representada por el punto de vista androcéntrico.

Aportando a las preguntas sobre quién puede ser sujeto de conocimiento, las pruebas a las que deben someterse las creencias para ser legitimadas como conocimiento y qué tipo de cosas pueden ser objeto de conocimiento (Harding, 2010), la epistemología feminista constituye "una excelente crítica tanto al relativismo como al puro perspectivismo, al situar la objetividad en el reconocimiento del punto de partida del propio conocer" (Fígari, 2011). Contra los principios aristotélicos de la realidad que fundan a la vez el mundo y la percepción del mundo (identidad, exclusión y no contradicción) (Maffia, 2011), concibe al mundo como sujeto activo cognoscible desde distintas posibilidades de relaciones sociales situadas, y apuesta a la activación de las categorías pasivas de objeto, a la 'implicación' desde una relación conversacional cargada de poder. Por último, al poner al objeto de conocimiento como sujeto activo, re-sitúa la ciencia social como discurso y aparato productor de significados y de cuerpos.

Desde la responsabilidad de la formación de un oficio teórico, práctico, emocional y político, para nosotras el hacer teoría implica "comprometernos activamente con (...) mundos, no tanto para hacer que se revelen de manera 'correcta', sino para hacer que existan y se muevan de manera diferente" (Flores, 2013, p. 27). A partir de estos desplazamientos epistémicos, entendemos que en el hacer investigación se modifican puntos de subjetivación propia y se ponen en cuestión jerarquías naturalizadas en los modos mismos de la enunciación.

Por ello, consideramos necesario oponer al automatismo de las tecnologías de la investigación una politica de las pedagogías en la que la construcción del oficio transmute en un devenir de prácticas no clausuradas y un re-conocimiento mutuo con quienes hacemos investigación. La persistente actitud de sospecha de lo conocido y de creatividad respecto de los dispositivos de los que nos valemos para "producir" lo que consideramos un dato social nos sitúa ya no por fuera de aquello que deseamos comprender, sino como parte de esto que deseamos subvertir.

\section{Contra los automatismos de la Programación metodológica: DeSEO-Placer- JUEGO EN LA EXPERIENCIA DE PRODUCIR CONOCIMIENTO}

"Existe politicidad ahí donde operan codificaciones de poder susceptibles de ser interrumpidas y
desviadas mediante actos críticos de oposición que subviertan sus jerarquías de valor y distinción,
sus normas autoritarias y sus totalizaciones represivas". sus normas autoritarias y sus totalizaciones represivas”.
Nelly Richard

¿Acaso no es la ciencia social una forma de discurso que piensa y construye sobre lo real? ¿Acaso no se juegan en la escritura subversiones contra decires universales? ¿Acaso no disputan, en ese juego, dispositivo y líneas de fuga, la potencia y lo real como concreto de múltiples y jerarquizadas determinaciones?

En el devenir del SFI tomamos como opción pedagógica presentar y ejercer la investigación como un oficio que humaniza y politiza el quehacer sociológico con rigurosidad creativa. Un oficio que se inscribe en la praxis misma, en los cuerpos de quienes investigamos, pero también de quienes aportan a nuestras investigaciones desde lo que entendemos y denominamos como un proceso ético de co-construcción de conocimiento. Se trata de un oficio que, lejos de pensarlo destinado a unes poques, es perfectible, acumulable, permeable a nuevos y permanentes desafíos del deseo personal y político y, sobre todo, encarnable; guiado por una dimensión ético-política que se opone a la moral. Proponemos una ética deleuziana que es afín a lo que incrementa las pasiones alegres y las potencia, unida a un oficio cuyo objetivo es la comprensión y el re-conocimiento de lo hasta entonces incomprendido, negado, des-conocido; que es a la vez productor y producido, objeto de permanente análisis crítico para no devenir "cosa".

Desde allí batallamos contra el positivismo neutral y avalorativo. Las herramientas que nos da el triple giro hermenéutico nos permiten pivotear en una crítica abierta, de final incierto, pero potentemente productiva 
de líneas de fuga hacia saberes co-construidos desde los criterios de proximidad histórica, relacionamiento implicado y perspectiva situada.

En este sentido, somos conscientes de que las metodologías ejercen un poder disciplinario que nos constituye por formación, y que intentamos subvertir subjetiva y colectivamente hacia líneas de fuga creativas, manteniendo una posición de vigilancia y alerta respecto del automatismo de sus procedimientos. Las técnicas de investigación, en tanto tecnologías de producción de saberes que se han consolidado a partir de la imposición del "argumento técnico" (Marradi, Archenti y Piovani, 2010, p. 12), prescriben procedimientos de investigación social frente a los cuales asumirse como investigadores implica la encarnación de la "higiene metodológica" de estas prácticas nunca abiertas, nunca inciertas.

Contra toda higiene del discurso metodológico y técnico, para nosotras la investigación es una apuesta hacia una auto-reflexividad política, sistemática y colectivizada sobre las prácticas del conocer-comprender, que proporcione elementos críticos para el uso coherente de las categorías y esquemas de pensamiento que identifiquen y disputen sentido a las formas instituidas y/o naturalizadas. Por este motivo, no promovemos recetas para la investigación, sino que -ya conocidas las prescripciones básicas de la metodología científica hegemónica- las estrategias se van construyendo artesanalmente en función de interrogantes que abren un campo de investigación, de interpelación subjetiva, teórica, política y metodológica.

Para producir quiebres en la maquinaria académica de producción de saberes individualizantes, heteropatriarcales, nos proponemos como inicio de la producción social de conocimiento un juego que está más allá del control de los métodos que encorsetan los posicionamientos teórico-políticos, y nos acerca a lo que Preciado (2019, p. 43) denomina "alianzas sintéticas": la constitución de grupos de investigación conformados por la diversidad de quienes están cursando la materia. El devenir en la construcción del grupo se inicia con la tensa puesta en común de los recorridos e intereses individuales de investigación de sus tres, máximo cuatro, integrantes. Aquí el grupo se constituye en espacio de debate, disputa y construcción de un abordaje posible, aunque nunca clausurado. Su elaboración implica hacerse cargo de las afectaciones desde el deseo individual en sentido deleuziano: "como una disposición, y no una carencia, como un proceso y no una estructura” (Manada de lobxs, 2014, pp. 43-44). Esta dinámica nos permite distanciarnos de los mecanismos de producción de la subjetividad del oficio hegemónico, abriendo paso a la creatividad por proximidad y alteridad, como momentos de tensión y flujo intermitentes en una misma construcción.

En ese juego de contrapuntos necesario que se mantiene a lo largo de todo el trabajo, un segundo momento fundante se da cuando quienes investigan se encuentran en proximidad y alteridad con quienes integran el campo de indagación. El trabajo en grupos se constituye en una posibilidad de encuentro en puntos de vista situados, que aportan a una construcción de miradas que expandan las posibilidades de decir, ver y habitar "lo real". En palabras de Haraway, "la lucha política consiste en ver desde las dos perspectivas a la vez, ya que cada una de ellas revela al mismo tiempo tanto las dominaciones como las posibilidades inimaginables desde otro lugar estratégico" (1984, p. 8).

\section{El PASAJE DE LaS FUgas: La INTERdEPENDENCIA RELACIONAL COMO VALIDACión}

Este momento nos conduce a una pregunta que dejamos planteada al inicio de la reflexión: la pregunta por la validez del conocimiento que producimos en esta forma de construcción. Podemos empezar por retomar la distinción clásica de Zetterberg (1981) entre validez externa e interna, que nos parece interesante diseccionar. La validez interna de un proceso de investigación estará dada, para este autor, en el progresivo nivel de adecuación y ajuste entre la teoría y las técnicas de investigación, y viceversa. La validez externa de una investigación, por su parte, se establece a partir de la relación empírica de la construcción teórica con aquello que intenta explicar: la determinación de la validez externa consiste en poner a prueba una hipótesis (Zetterberg, 1981, p. 96). 
Por su parte, Howe y Eisenhart sostienen que la cuestión de los criterios para valorar la investigación debe abordarse desde la lógica en uso asociada a diversas metodologías de investigación: "dada la imposibilidad de una unidad monolítica del método científico (la que surgiría de los sueños del positivismo) los criterios deben anclarse dentro del proceso de investigación" (en Sandín Esteban, 2000, p. 177). Aquí, el significado tradicional del concepto de validez ha sido reformulado, fundamentalmente, en términos de construcción social del conocimiento, otorgando un nuevo énfasis a la interpretación (2000, p. 226).

En nuestra propuesta abdicamos del positivismo que concibe la doble existencia de la validación y nos reconocemos subjetividades involucradas en el proceso de enunciación y producción de sentidos con otres sobre fragmentos no azarosos de ese mundo. En ese sentido, nos reapropiamos de la validez interna como progresivo ajuste entre las herramientas conceptuales que elegimos provisionalmente necesarias y una práctica ético-política desde la que hacemos uso de ellas en un encuentro con otres.

Asimismo, desde las alianzas sintéticas con quienes integran "el campo", sus experiencias, sentidos y creatividad, tejemos aproximaciones por la vía de la "resonancia intensiva" (Rolnik, 2019). Desde allí estas aportan y le dan a lo producido validación "externa” en tanto co-construcción social. Recuperamos ambos aspectos -indisociables- de la validación ético-política que produce un trabajo grupal abierto, desde la proximidad histórica y la resonancia intensa que constituye el punto de partida de esas alianzas.

Por último, hacemos hincapié en lo que convencionalmente conocemos como el momento del análisis, en tanto instancia de re-construcción teórica anclada en la experiencia, como momento de diálogo colectivo hecho escritura, con las preguntas que iniciaron el camino de indagación. Escritura mutante, selectiva en la consideración de emergentes y potente en la intencionalidad política de los conocimientos que presenta. Momento particular del proceso de investigación, en el que señalamos la exposición como política de integración textual de la experiencia, en un formato abierto, siempre revisable, en una producción artística con intencionalidad política. La escritura como lugares situados de resistencia desde donde enriquecer y/o desentramar imaginarios hegemónicos consolidados, crear otras tramas posibles, otros códigos, otras reglas, otros modos de nombrar la historia y el presente.

Finalmente, sacar a la luz un proceso de investigación implica para nosotras una apuesta de sentido.

"La demarcación simbólica de lo social crea límites entre lo permitido y lo prohibido, lo excluido y lo integrado, lo correcto y lo incorrecto, lo posible y lo imposible y está fuertemente arraigada en la tradición, las costumbres y, por ello, también en las disciplinas académicas. (...) Para ampliar lo vivible consideramos fundamental ampliar lo visible; (...) abrir pasaje a genealogías otras, voces silenciadas, experiencias invisibilizadas; saturar el ojo social de la producción de nuevos sentidos, imágenes, conceptos, (...) con documentos que configuren una nueva tradición de la discontinuidad, la pluralidad, y lo imprevisible" (Sentamans, 2013, pp. 36-37).

Está claro que visibilidad significa existencia y la producción de nuevos sentidos abre una puerta a la construcción no normalizadora del oficio, disruptiva, creativa, placentera, íntimamente entrelazada con lo que produce como relaciones y significados. Buscamos producir textos que emerjan como acontecimiento, "instrumentos que permitan diseccionar y proyectar luz sobre regiones oscuras de la vida social que se podrían leer como espacios para pensar nuevas dimensiones de la reflexión y la praxis política” (Solá, 2013, p. 24)

\section{Cierres iN-esperados}

"Pensar ni consuela ni hace feliz. Pensar se arrastra lánguidamente como una perversión; pensar se
repite con aplicación sobre un teatro; pensar se echa de golpe fuera del cubilete de los dados. Y
cuando el azar, el teatro y la perversión entran en resonancia, cuando el azar quiere que entre los
tres haya esta resonancia, entonces el pensamiento es un trance; y entonces vale la pena pensar."
Michel Foucault, "Theatrum Philosophicum"

En nuestros devenires, los que son, los que están siendo, un oficio es poner cuerpo, hacer con otres, en movimiento, en la proximidad. La pregunta no es cómo se llega a ser investigadoras sino cómo nos hacemos 
y hacemos a otres co-productores de un nuevo orden simbólico. Para hacer inteligibles las estrategias del poder, para mostrar los artificios de lo instituido, para visibilizar imágenes que alimenten nuestros deseos de transformación.

Desde miradas situadas, que sostienen la necesidad de aplicar el análisis a las prácticas que desarrollamos como investigadoras en nuestra tarea cotidiana, disputamos al saber hegemónico académico formas de hacer posible la reflexión teórica de manera in-disciplinada, en el ejercicio de mirar desde los márgenes y desde la singularidad en relación con otres.

Luchar contra los sistemas hegemónicos de regulación del deseo, de cuerpos e identidades, de saberes y procedimientos abriendo paso a otras experiencias para la producción de sentidos es imaginar e inscribir nuevas prácticas sociales para la investigación y el oficio. Nos motiva la necesidad de re-politizar la práctica investigativa, descentralizar el conocimiento teórico, colmar de significado el oficio, sus preguntas y sus respuestas, quitándole su andar autómata de "receta" y reivindicando la inquietud por la experimentación. Desbordar nuestras prácticas de intensidad y sentido, como modo de ampliar los límites, haciendo así evidente su artificio naturalizado, fugarse del oficio autómata hacia opciones de mutuo re-conocimiento creativo.

\section{ReFERENCIAS}

Berkin, S. y Kaltmeier, O. (2012). En diálogo. Metodologías horizontales en ciencias sociales y culturales. México D. F.: Gedisa.

Bourdieu, P. (2002). El oficio de sociólogo. Buenos Aires: Siglo XXI editores.

Figari, C. (2010). Conocimiento situado y técnicas amorosas de la ciencia. Tópicos de epistemología crítica. Recuperado de https://epistemologiascriticas.files.wordpress.com/2011/05/figari_conoc-situado.pdf

Flores, val. (2013). Interruqciones. Buenos Aires: La mondonga dark ediciones.

Foucault, M. (1968). Prólogo “Theatrum philosophicum”. En G. Deleuze, Repetición y diferencia (pp. 7-40). Barcelona: Anagrama.

Foucault, M. (1988). El sujeto y el poder. Revista Mexicana de Sociología, 50(3), 3-20.

Gago, V. y Obarrio, J. (2016). Prólogo. En A. Mbembé Crítica de la razón negra (pp. 6-19). Buenos Aires: Futuro anterior ediciones.

Haraway, D. (1984). Manifiesto Ciborg. El sueño irónico de un lenguaje común para las mujeres en el circuito integrado. Madrid: Puente aéreo ediciones.

Haraway, D. (1995). Ciencia, cyborgs y mujeres. La reinvención de la naturaleza. Valencia: Ediciones Cátedra.

Harding, S. (2010). ¿Existe un método feminista? En Harding, S. Feminism and Methodology (pp. 9-34). Indianápolis: Indiana University Press.

Maffia, D. (2011). Sexo, género, diversidades y disidencias sexuales. Conferencia dictada en Universidad de San Andrés, septiembre. Buenos Aires, Argentina. https://www.youtube.com/watch?v=LFiIUr4Nzho\&t=1046s

Manada de Lobxs (2014). Foucault para encapuchadas. Buenos Aires: Milena Caserola.

Marradi, A., Archenti, N. y Piovani, J. I. (2010). El diseño de la investigación: la elección de un tema, la delimitación del problema y su operativización. En A. Marradi, N. Archenti y J.I. Piovani (Eds.), Metodología de las Ciencias Sociales (pp. 53-65). Buenos Aires: Cengage Learning.

Preciado, P. (2019). Un apartamento en Urano. Crónicas del cruce. Barcelona: Anagrama.

Rivera Cusicanqui, S. (2010). Sociología de la imagen: miradasch'ixi desde la historia andina. Buenos Aires: Tinta limón.

Rolnik, S. (2019). Esferas de la insurrección. Apuntes para descolonizar el inconsciente. Buenos Aires: Tinta Limón.

Sánchez Rubio, D. (2012). Prólogo. En M. Díaz y C. Pescader (Comps.), Descolonizar el presente: ensayos críticos desde el Sur (pp. 9-18). Río Negro: Publifadecs, Universidad Nacional del Comahue. 
Sandín Esteban, M. P. (2000). Criterios de validez en la investigación cualitativa: de la objetividad a la solidaridad. Revista de Investigación Educativa, 18(1) 223-242. Barcelona: Universidad de Barcelona. Recuperado de https ://bit.ly/2Udn3Kl

Scardamaglia. V. (2018). El trabajo universitario como acto de pensamiento. Intersecciones Psi, 8(27), s/p. Disponible en https://bit.ly/2IqeEjO

Segato, R. (2010a). Las estructuras elementales de la violencia. Buenos Aires: Prometeo.

Segato, R. (2010b). Género y colonialidad: en busca de claves de lectura y de un vocabulario estratégico descolonial. En A. Quijano y J. Mejía Navarrete (Eds.), La cuestión descolonial. Lima: Universidad Ricardo Palma.

Segato, R. (2013). La crítica de la colonialidad en ocho ensayos. Buenos Aires: Prometeo.

Sentamans, T. (2013). Redes transfeministas y nuevas políticas de representación sexual (I) Diagramas de flujos. En M. Solá y E. Urko (comps.), Transfeminismos. Epistemes, fricciones y fujos (pp. 35-44). Tafalla: Txalaparta ediciones.

Solá, M. (2013). Introducción: Textos, pretextos y contextos. En Solá, M. y E. Urko (comps.), Transfeminismos. Epistemes, fricciones y fujos (pp. 15-30). Tafalla: Txalaparta ediciones.

Zetterberg, H. (1981). Capítulo 7: Sobre las decisiones en los estudios verificativos. En Teoría y Verificación en Sociología (pp. 95-126). Buenos Aires: Nueva Visión. 